Geometry 85 Topology

Volume 7 (2003) 1-31

Published: 23 January 2003

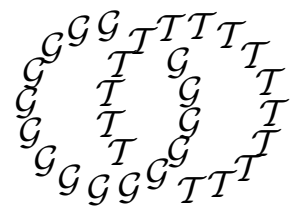

\title{
Two applications of elementary knot theory to Lie algebras and Vassiliev invariants
}

\author{
Dror BAR-NATAN \\ Dept of Mathematics \\ University of Toronto \\ Toronto ON M5S $3 G 3$ \\ Canada \\ Thang T Q LE \\ Dept of Mathematics \\ SUNY at Buffalo \\ Buffalo NY 14214 \\ USA \\ Dylan P Thurston \\ Dept of Mathematics \\ Harvard University \\ Cambridge, MA 02138 \\ USA \\ drorbn@math.toronto.edu letu@math.buffalo.edu dpt@math.harvard.edu \\ http://www.math.toronto.edu/ drorbn buffalo.edu/ letu harvard.edu/ dpt
}

\begin{abstract}
Using elementary equalities between various cables of the unknot and the Hopf link, we prove the Wheels and Wheeling conjectures of $[5,9]$, which give, respectively, the exact Kontsevich integral of the unknot and a map intertwining two natural products on a space of diagrams. It turns out that the Wheeling map is given by the Kontsevich integral of a cut Hopf link (a bead on a wire), and its intertwining property is analogous to the computation of $1+1=2$ on an abacus. The Wheels conjecture is proved from the fact that the $k$-fold connected cover of the unknot is the unknot for all $k$.

Along the way, we find a formula for the invariant of the general $(k, l)$ cable of a knot. Our results can also be interpreted as a new proof of the multiplicativity of the Duflo-Kirillov map $S(\mathfrak{g}) \rightarrow U(\mathfrak{g})$ for metrized Lie (super-)algebras $\mathfrak{g}$.
\end{abstract}

AMS Classification numbers Primary: 57M27

Secondary: 17B20, 17B37

Keywords: Wheels, Wheeling, Vassiliev invariants, Hopf link, $1+1=2$, Duflo isomorphism, cabling

Proposed: Vaughan Jones

Seconded: Yasha Eliashberg, Joan Birman
Received: 9 May 2002 Accepted: 8 November 2002 


\section{Introduction}

\subsection{The Duflo-Kirillov isomorphism}

The Duflo-Kirillov isomorphism is an algebra isomorphism between the invariant part of the symmetric algebra and the center of the universal enveloping algebra for any Lie algebra $\mathfrak{g}$. This isomorphism was first described for semisimple Lie algebras by Harish-Chandra. Kirillov gave a formulation of the Harish-Chandra map that has meaning for all finite-dimensional Lie algebras, and conjectured that it is always an algebra isomorphism. The conjecture was proved by Duflo [11]. Although the Kirillov-Duflo map can be formulated in a very explicit way as a linear map between two pretty simple algebras (with very explicit structure), all known proofs of the Duflo theorem were difficult: In the book of Dixmier [10], the proof is given only in the last chapter and it utilizes most of results developed in the whole book, including many classification results (a situation Godement [12] called "scandalous"). As discussed below, there have been several recent proofs that do not use classification results, but they all use tools from well outside the natural domain of the problem.

Let us review briefly the Duflo theorem. The Poincaré-Birkhoff-Witt map between the symmetric algebra and the universal enveloping algebra of a Lie algebra $\mathfrak{g}$,

$$
\chi: S(\mathfrak{g}) \longrightarrow U(\mathfrak{g}),
$$

given by taking a monomial $x_{1} \ldots x_{n}$ in $S(\mathfrak{g})$ and averaging over the product (in $U(\mathfrak{g})$ ) of the $x_{i}$ in all possible orders, is an isomorphism of vector spaces and $\mathfrak{g}$-modules. Since $S(\mathfrak{g})$ is abelian and $U(\mathfrak{g})$ is generally not, $\chi$ is clearly not an algebra isomorphism. Even restricting to the invariant subspaces on both sides,

$$
\chi: S(\mathfrak{g})^{\mathfrak{g}} \longrightarrow U(\mathfrak{g})^{\mathfrak{g}}=\text { center of } U(\mathfrak{g}),
$$

$\chi$ is still not an isomorphism of algebras.

The Duflo theorem says that the combination $\chi \circ \partial_{j^{\frac{1}{2}}}$, with $\partial_{j^{\frac{1}{2}}}: S(\mathfrak{g}) \longrightarrow S(\mathfrak{g})$ defined below, is an algebra isomorphism between $S(\mathfrak{g})^{\mathfrak{g}}$ and $U(\mathfrak{g})^{\mathfrak{g}}$.

Here $j^{\frac{1}{2}}(x)$ is a formal power series (beginning with 1 ) on $\mathfrak{g}$, defined by

$$
j^{\frac{1}{2}}(x)=\operatorname{det}^{\frac{1}{2}}\left(\frac{\sinh \left(\frac{1}{2} \operatorname{ad} x\right)}{\frac{1}{2} \operatorname{ad} x}\right) .
$$

The operator $\partial_{j^{\frac{1}{2}}}$ is obtained by plugging the (commuting) vector fields $\partial / \partial x^{*}$ (on $\mathfrak{g}^{*}$ ) in the power series $j^{\frac{1}{2}}$. (Note that for $x^{*} \in \mathfrak{g}^{*}, \partial / \partial x^{*}$ transforms like 
an element of $\mathfrak{g})$. The result is an infinite-order differential operator on $\mathfrak{g}^{*}$, which we can then apply to a polynomial on $\mathfrak{g}^{*}$ (三 an element of $S(\mathfrak{g})$ ). For details, see [11]. The function $j^{\frac{1}{2}}(x)$ plays an important role in Lie theory. Its square, $j(x)$, is the Jacobian of the exponential mapping from $\mathfrak{g}$ to the Lie group $G$. The operator $\partial_{j^{\frac{1}{2}}}$ is called the strange isomorphism by Kontsevich $[16]$.

\section{$1.2 \quad$ Elementary knot theory}

We will touch upon two simple facts in knot theory that have deep consequences for Lie algebras and Vassiliev invariants. The two facts can be summarized by the catch phrases " $1+1=2$ " and " $n \cdot 0=0 . "$

- " $1+1=2$." This refers to a fact in "abacus arithmetic." On an abacus, the number 1 is naturally represented by a single bead on a wire, as in Figure 1(a), which we think of as a tangle. The fact that $1+1=2$ then becomes the equality of the two tangles in Figure 1(b). On the left side of the figure, " $1+1$ ", the two beads are well separated, as for connect sum of links or multiplication of tangles; on the right side, "2", we instead start with a single bead and double it, so the two beads are very close together.

In other terms, the connected sum of two Hopf links is the same as doubling one component of a single Hopf link, as in Figure 1(c).

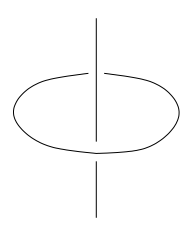

(a) The link " 1 "
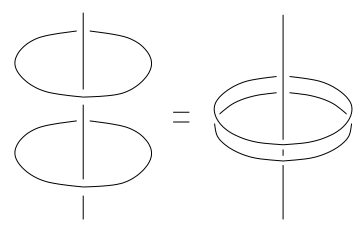

(b) $" 1+1=2 "$

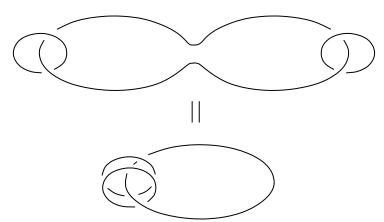

(c) An alternate version of $" 1+1=2 "$

Figure 1: Elementary knot theory, part 1

- " $n \cdot 0=0$." In the spirit of abacus arithmetic, 0 is represented as just a single vertical strand. We prefer to close it off, yielding the knot in Figure 2(a). The knot $n \cdot 0$ is then this knot repeated $n$ times, as in Figure 2(b). The two knots are clearly the same, up to framing. 


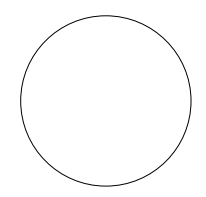

(a) The knot "0"

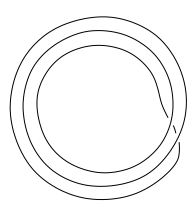

(b) The knot " $n \cdot 0$, " shown here for $n=3$

Figure 2: Elementary knot theory, part 2

\subsection{Wheels and wheeling: main results}

The bridges between the knot theory of Section 1.2 and the seemingly quite disparate Lie algebra theory of Section 1.1 are a certain spaces of uni-trivalent diagrams (called Jacobi diagrams) modulo local relations. (See Section 2.1, the 1-valent vertices are called the "legs" of the diagram.) On the one hand, such diagrams give elements of $U(\mathfrak{g})$ or $S(\mathfrak{g})$ for every metrized Lie algebra $\mathfrak{g}$ in a uniform way; on the other hand, they occur naturally in the study of finite type invariants of knots [3, 15]. Like the associative algebras $S(\mathfrak{g})$ and $U(\mathfrak{g})$ associated to Lie algebras, these diagrams appear in two different varieties: $\mathcal{A}$, in which the legs have a linear order, as in Figure $3(\mathrm{a})$, and $\mathcal{B}$, in which the legs are unordered, as in Figure 3(b). As for Lie algebras, they each have a natural algebra structure (concatenation and disjoint union, respectively); and there is an isomorphism $\chi: \mathcal{B} \rightarrow \mathcal{A}$ between the two (averaging over all possible orders of the legs).

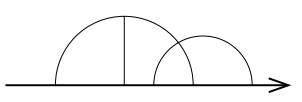

(a) A sample element of $\mathcal{A}$

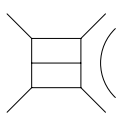

(b) A sample element of $\mathcal{B}$

Figure 3: Examples of Jacobi diagrams

There is one element of the algebra $\mathcal{B}$ that will be particularly important for us: the "wheels" element. It is the diagrammatic analogue of the function $j^{\frac{1}{2}}$ above:

$$
\Omega=\exp \sum_{n=1}^{\infty} b_{2 n} \omega_{2 n} \in \mathcal{B}
$$


where:

- The 'modified Bernoulli numbers' $b_{2 n}$ are defined by the power series expansion

$$
\sum_{n=0}^{\infty} b_{2 n} x^{2 n}=\frac{1}{2} \log \frac{\sinh x / 2}{x / 2} .
$$

These numbers are related to the usual Bernoulli numbers $B_{2 n}=4 n$. $(2 n) ! \cdot b_{2 n}$ and to the values of the Riemann $\zeta$-function on the even integers. The first three modified Bernoulli numbers are $b_{2}=1 / 48$, $b_{4}=-1 / 5760$, and $b_{6}=1 / 362880$.

- The ' $2 n$-wheel' $\omega_{2 n}$ is the degree $2 n$ Jacobi diagram made of a $2 n$-gon with $2 n$ legs:

$$
\omega_{2}=\circlearrowleft, \quad \omega_{4}=\square\left(, \omega_{6}=Y, \ldots\right.
$$

Let $\partial_{\Omega}$ be the operation of applying $\Omega$ as a differential operator, which takes a diagram $D$ and attaches some of its legs to all the legs of $\Omega$. (See Section 2.5 for the precise definition.)

The first main result of this paper is the following analog of the Duflo theorem.

Wheeling Theorem The map $\Upsilon=\chi \circ \partial_{\Omega}: \mathcal{B} \rightarrow \mathcal{A}$ is an algebra isomorphism.

Although the Wheeling theorem was motivated by Lie algebra considerations when it was first conjectured $[5,9]$, the proof we will give, based on the equation " $1+1=2$ " from Section 1.2, is entirely independent of Lie algebras and is natural from the point of view of knot theory. In particular, we obtain a new proof of the Duflo theorem for metrized Lie algebras, with some advantages over the original proofs by Harish-Chandra, Duflo, and Cartan: our proof does not require any detailed analysis of Lie algebras, and so works in other contexts in which there is a Jacobi relation. For instance, our proof works for super Lie algebras with no modification.

The Wheeling theorem has already seen several applications. We will use it to compute the Kontsevich integral of the unknot, using our second elementary knot theory identity " $n \cdot 0=0$ ".

Wheels Theorem The Kontsevich integral of the unknot is

$$
Z(\bigcirc)=\Omega \in \mathcal{B} \text {. }
$$


The Wheeling theorem was first conjectured by Deligne [9] and by Bar-Natan, Garoufalidis, Rozansky and Thurston [5], who also conjectured the Wheels theorem.

Along the way we also find a formula describing the behaviour of the Kontsevich integral under connected cabling of knots. We also compute the Kontsevich integral of the Hopf link $Q$; which is intimately related to the map $\Upsilon$ above.

Further computations for a sizeable class of knots, links, and 3-manifolds (including all torus knots and Seifert-fiber homology spheres) have been done by Bar-Natan and Lawrence [7]. Hitchin and Sawon [14] have used the Wheeling theorem to prove an identity expressing the $L^{2}$ norm of the curvature tensor of a hyperkähler manifold in terms of Pontryagin classes. In a future paper [25] one of us (DPT) will show how to write simple formulas for the action of $\mathfrak{s} l_{2}(\mathbb{Z})$ on the vector space associated to a torus in the perturbative TQFT of $\mathrm{Mu}-$ rakami and Ohtsuki [24]. Our connected cabling formula also finds application in recent work of Roberts and Willerton on the "total Chern class" invariant of knots.

There are two other recent proofs of the Wheeling theorem. One is due to Kontsevich [16, Section 8], as expanded by [2, 13, 23]. Kontsevich's proof is already at a diagrammatic level, similar to the one in this paper, although it is more general: it works for all Lie algebras, not just metrized ones. His proof again uses a transcendental integral, similar in spirit to the "Kontsevich integral" in the theory of Vassiliev invariants [15]. Another proof is due to Alekseev and Meinrenken [1]. The Alekseev and Meinrenken paper is not written in diagrammatic language, but seems to extend to the diagrammatic context without problems. Their proof does not involve transcendental integrals: the only integral in their proof is in the proof of the Poincaré lemma (the homology of $\mathbb{R}^{n}$ is trivial in dimension $>0$ ).

\subsection{Plan of the paper}

In the first section we review the theory of Jacobi diagrams. Section 3 is devoted to cabling formulas of the Kontsevich integral which are crucial in the proofs of main theorems. In Sections 4 and 5 we prove the Wheeling and Wheels theorems. In Section 6 we calculate the values of the Kontsevich integral of the Hopf link. In the Appendix we give a self-contained method to determine the coefficients of the wheels element. 


\subsection{Acknowledgement}

Research by the authors DBN and DPT was supported in part by BSF grant \#1998-119. The author TTQL was partially supported by NSF grant DMS9626404 and a postdoc fellowship at the Mathematical Sciences Research Institute in Berkeley in 1996-1997. Research at MSRI was supported in part by NSF grant DMS-9022140. The author DPT was supported by an NSF Graduate Student Fellowship, a Sloan Dissertation Fellowship, and an NSF Postdoctoral Research Fellowship. In addition we wish to thank A Haviv, J Lieberum, A Referee and J Roberts for their comments and suggestions.

\section{Preliminaries on Jacobi diagrams}

We recall basic definitions and some known properties of Jacobi diagrams in this section. For details, see [3].

\subsection{Jacobi diagrams}

An open Jacobi diagram (sometimes called a Chinese Character, uni-trivalent graph, or web diagram) is a vertex-oriented uni-trivalent graph, ie, a graph with univalent and trivalent vertices together with a cyclic ordering of the edges incident to the trivalent vertices. Self-loops and multiple edges are allowed. A univalent vertex is called a leg, and trivalent vertex is also called an internal vertex. In planar pictures, the orientation on the edges incident on a vertex is the clockwise orientation, unless otherwise stated. The degree of an open Jacobi diagram is half the number of vertices (trivalent and univalent). Some examples are shown in Figure 3(b).

Suppose $X$ is a compact oriented 1-manifold (possibly with boundary, often with labeled components) and $Y$ a finite set of (labeled) asterisks, symbols of the form $*_{x}, *_{y}$, etc.. A Jacobi diagram based on $X \cup Y$ is a graph $D$ together with a decomposition $D=X \cup \Gamma$, where $\Gamma$ is an open Jacobi diagram with some legs labeled by elements of $Y$, such that $D$ is the result of gluing all the non-labeled legs of $\Gamma$ to distinct interior points of $X$. Note that repetition of labels is allowed, and not all labels have to be used. The degree of $D$, by definition, is the degree of $\Gamma$. Usually $X$ is called the skeleton of $D$, and in picture is depicted by bold lines.

Suppose $\phi: X^{\prime} \rightarrow X$ is a covering map between compact oriented 1-manifolds, and $D=X \cup \Gamma$ is a Jacobi diagram based on $X \cup Y$. The pull-back $\phi^{*}(D)$ is 
the sum over all Jacobi diagrams $D^{\prime}$ based on $X^{\prime} \cup Y$ such that $\phi\left(D^{\prime}\right)=D$. Here $\phi\left(D^{\prime}\right)=D$ means $D^{\prime}=X^{\prime} \cup \Gamma$ and $\phi$ can be extended to $D^{\prime}$ so that it is identity on $\Gamma$.

The space $\mathcal{A}^{f}(X \cup Y), X$ and $Y$ as above, is the space of Jacobi diagrams based on $X \cup Y$ modulo the usual antisymmetry, IHX and STU relations (see [3]). The completion of $\mathcal{A}^{f}(X \cup Y)$ with respect to degree is denoted by $\mathcal{A}(X \cup Y)$. When $\phi: X^{\prime} \rightarrow X$ is a cover, the pull-back $\phi^{*}$ descends to a well-defined map from $\mathcal{A}(X \cup Y)$ to $\mathcal{A}\left(X^{\prime} \cup Y\right)$. An example of pull-backs is the Adams operation in $[3]$.

Let $\mathcal{A}^{\mathrm{bc}}(X \cup Y)$ be the subspace of $\mathcal{A}(X \cup Y)$ spanned by boundary-connected Jacobi diagrams: diagrams with no connected components that are disjoint from the skeleton $X$.

There is a natural map from $\mathcal{A}(\uparrow \cup X)$ to $\mathcal{A}(\circlearrowright \cup X)$ given by attaching the two endpoints of the interval $\uparrow$. If $X$ is a closed 1-manifold, then this map is an isomorphism. In particular, when $X=\emptyset$, the spaces $\mathcal{A}(\uparrow)$ and $\mathcal{A}(\circlearrowright)$ are canonically isomorphic. But this is not true if $X$ has an interval component. Explicitly, $\mathcal{A}(\uparrow \uparrow) \not \mathcal{A}(\uparrow \circlearrowright) \simeq \mathcal{A}(\circlearrowright \circlearrowright)$.

An open Jacobi diagram is strutless if it does not have a connected component homeomorphic to a strut $\frown$, ie an interval. A strutless element of $\mathcal{A}(Y)$, where $Y$ is a set of asterisks, is a linear combination of strutless diagrams.

\subsection{Special interesting cases}

Of special interest are the following $\mathcal{A}(X \cup Y)$.

For $X=\emptyset$ and $Y$ has one element, the space $\mathcal{A}(X \cup Y)$ is denoted by $\mathcal{B}$. Note that all the labels of legs of diagrams in $\mathcal{B}$ are the same, and we often forget the labels. There is a natural product in $\mathcal{B}$ defined by taking disjoint union of diagrams. With this product $\mathcal{B}$ is a commutative algebra. The wheels $\omega_{2 n}$ introduced in the introduction belongs to $\mathcal{B}$.

For $X=\circlearrowright$, the oriented circle, and $Y=\emptyset$, the space $\mathcal{A}(\circlearrowright)$, also denoted simply by $\mathcal{A}$, is the space in which lie the values of the Kontsevich integral of a knot. There is a natural product in $\mathcal{A}$ defined by taking connected sums of diagrams based on $\circlearrowright$. With this product $\mathcal{A}$ is a commutative algebra. As noted before, $\mathcal{A}$ is canonically isomorphic to $\mathcal{A}(\uparrow)$, and we will often identify these vector spaces. Note that the space $\mathcal{A}$ of $[3,20]$ is equal to our $\mathcal{A}^{\mathrm{bc}}(\circlearrowright)$, the boundary-connected part. 
Suppose $X=Y=\emptyset$. The space $\mathcal{A}(\emptyset)$ is the space in which lie the values of the LMO invariants of 3-manifolds [21]. With disjoint union as the product, $\mathcal{A}(\emptyset)$ becomes a commutative algebra, and all other $\mathcal{A}(X \cup Y)$ have a natural $\mathcal{A}(\emptyset)$-module structure.

It is known that for any metrized Lie algebra $\mathfrak{g}$, there are the weight maps, which are algebra homomorphisms, $W_{\mathfrak{g}}: \mathcal{B}^{f} \rightarrow S(\mathfrak{g})^{\mathfrak{g}}$ and $W_{\mathfrak{g}}: \mathcal{A}^{f}(\circlearrowright) \rightarrow U(\mathfrak{g})^{\mathfrak{g}}$, see [3]. Here $S(\mathfrak{g})$ and $U(\mathfrak{g})$ are respectively the symmetric algebra and the universal enveloping algebra of $\mathfrak{g}$, and $M^{\mathfrak{g}}$ is the invariant subspace of the $\mathfrak{g}$ module $M$. Thus $U(\mathfrak{g})^{\mathfrak{g}}$ is the center of $U(\mathfrak{g})$. In some sense, one can think of $\mathcal{A}$ and $\mathcal{B}$ as being related to a "universal (metrized) Lie algebra", incorporating information about all Lie algebras at once. But $\mathcal{B}$ and $\mathcal{A}$ are both bigger and smaller than that. For example, the map from $\mathcal{B}$ to the product of $S(\mathfrak{g})^{\mathfrak{g}}$ for all metrized Lie algebras is neither injective nor surjective: There are elements of $\mathcal{B}$ that are non-zero but become zero when evaluated in any metrized Lie algebra $[26,22]^{1}$. Not all elements of $S(\mathfrak{g})^{\mathfrak{g}}$ are in the image of the map $W_{\mathfrak{g}}$. (For instance, the image of $W_{\mathfrak{g}}$ consists of polynomials of even order only.)

\subsection{Symmetrization maps}

One can define an analog of the Poincare-Birkhoff-Witt isomorphism for diagrams as follows.

Suppose $X$ is a collection of compact oriented 1-manifolds and asterisks. The symmetrization map $\chi_{x}: \mathcal{A}\left(*_{x} \cup X\right) \rightarrow \mathcal{A}\left(\uparrow_{x} \cup X\right)$ is a linear map defined on a diagram $D$ by taking the average over all possible ways of ordering the legs labeled by $x$ and attach them to an oriented interval. It is known that $\chi_{x}$ is a vector space isomorphism [3].

In particular, the symmetrization map $\chi: \mathcal{B} \rightarrow \mathcal{A}(\uparrow) \equiv \mathcal{A}(\circlearrowright)$ is an isomorphism of vector spaces, but it is not an algebra isomorphism. We drop the label here. The two products, disjoint union and connected sum, live on isomorphic spaces $\mathcal{B}$ and $\mathcal{A}$, and may be confused. We usually write out the product in cases of ambiguity.

\footnotetext{
${ }^{1}$ These references only deal with semi-simple Lie (super-) algebras, but according to Vogel and Lieberum (via private communications), Vogel's results extend to all metrized Lie (super-) algebras.
} 


\subsection{Symmetrization for closed components of the skeleton}

We have seen that, using the symmetrization map, one can trade an oriented interval in $X$ with an asterisk. We want to do the same with closed component in $X$. For this we need the link relations.

Suppose $*_{y}$ is an element of $Y$. If a leg of a diagram is labeled $y$, then the edge having this leg as an end is called a y-edge. In $\mathcal{A}(X \cup Y)$, link relations on $y$ are parametrized by Jacobi diagrams based on $X \cup Y$ in which one of the $y$-labeled legs is distinguished. The corresponding link relation is the sum of all ways of attaching the distinguished leg to all the other $y$-edges:

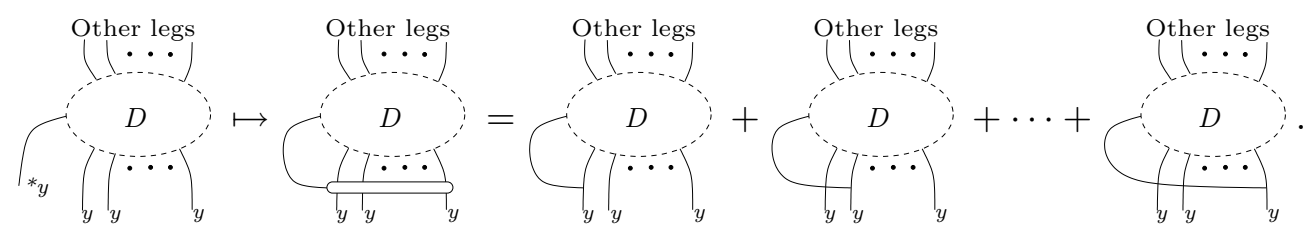

Suppose $X$ is a compact oriented 1-manifold, $Y$ is a set of asterisks $*$, and $Y^{\prime}$ is a set of circled asterisks, symbols of the form $\circledast_{x}, \circledast_{y}$, etc. Define $\mathcal{A}\left(X \cup Y \cup Y^{\prime}\right)$ as the space of Jacobi diagrams based on $X \cup Y \cup Y^{\prime}$ modulo the anti-symmetry, IHX, and STU relations as before and, in addition, link relations on each label in $Y^{\prime}$.

Suppose a circled asterisk $\circledast_{y}$ is not in $Y^{\prime}$. The symmetrization map $\chi_{x}: \mathcal{A}\left(\circledast_{y} \cup\right.$ $\left.X \cup Y \cup Y^{\prime}\right) \rightarrow \mathcal{A}\left(\circlearrowright_{y} \cup X \cup Y \cup Y^{\prime}\right)$ is the linear map defined on a diagram $D$ by taking the average over all possible ways of cyclic-ordering the legs labeled by $y$ and attach them to the circle $\circlearrowright_{y}$. It is known that $\chi_{y}$ is a vector space isomorphism [6].

\subsection{Diagrammatic Differential Operators}

For a strutless diagram $C \in \mathcal{B}$, the operation of applying $C$ as a differential operator, denoted $\partial_{C}: \mathcal{B} \rightarrow \mathcal{B}$, is defined to be

$$
\partial_{C}(D)= \begin{cases}0 & \text { if } C \text { has more legs than } D, \\ \text { the sum of all ways of gluing all } & \\ \text { the legs of } C \text { to some (or all) legs } & \text { otherwise. } \\ \text { of } D & \end{cases}
$$

For example,

$$
\partial_{\omega_{4}}\left(\omega_{2}\right)=0 ; \quad \partial_{\omega_{2}}\left(\omega_{4}\right)=80
$$


One might think of $D$ as a monomial of degree equal to the number of legs. If $C$ has $k$ legs and degree $m$, then $\partial_{C}$ is an operator of degree $m-k$. By linear extension, we find that every strutless $C \in \mathcal{B}$ defines an operator $\partial_{C}: \mathcal{B} \rightarrow \mathcal{B}$. (We restrict to diagrams without struts to avoid circles arising from the pairing of two struts and to guarantee convergence: gluing with a strut lowers the degree of a diagram, and so the pairing would not extend from $\mathcal{B}^{f}$ to $\mathcal{B}$.)

In some sense, $\partial_{C}$ is a diagrammatic analogue of a constant coefficient differential operator. For instance, one has:

- A diagram $C$ with $k$ legs reduces the number of legs by $k$, corresponding to a differential operator of order $k$.

- If $k=1$ ( $C$ has only one leg), we have a Leibniz rule like that for linear differential operators:

$$
\partial_{C}\left(D_{1} \sqcup D_{2}\right)=\partial_{C}\left(D_{1}\right) \sqcup D_{2}+D_{1} \sqcup \partial_{C}\left(D_{2}\right) .
$$

(Actually, all diagrams with only one leg are 0 in $\mathcal{B}$, so we have to extend our space of diagrams slightly for this equation to be non-empty. Adding some extra vertices of valence 1 satisfying no relations is sufficient.)

- Multiplication on the differential operator side is the same thing as composition:

$$
\partial_{C_{1} \sqcup C_{2}}=\partial_{C_{1}} \circ \partial_{C_{2}}
$$

\section{Cabling}

The behaviour of cabling will be crucial to the proofs of all of the Theorems of this paper. In this section, we will review some results of [20] on disconnected cabling and prove a new result on connected cabling.

\subsection{Tangles, framed tangles, and the Kontsevich integral}

Suppose $X$ is a compact oriented 1-manifold. A tangle with skeleton $X$ is a smooth proper embedding of $X$ into $\mathbb{R} \times \mathbb{R} \times[0,1] \subset \mathbb{R}^{3}$, considered up to isotopy relative to the boundary. The Kontsevich of such a tangle takes value in the space $\mathcal{A}^{\prime}(X)$, obtained from $\mathcal{A}(X)$ by dividing by the framing independence relation which says that a diagram containing an isolated chord is equal to 0 (see [3], we will not need $\mathcal{A}^{\prime}(X)$ in the future). When $X$ does not have any circle component, there is a canonical embedding from $\mathcal{A}^{\prime}(X)$ into $\mathcal{A}(X)$, and the Kontsevich integral can be considered valued in $\mathcal{A}(X)$. 
The framed Kontsevich integral of a framed tangle with skeleton $X$ takes value in $\mathcal{A}(X)$ (no framing independence relation here). For technical reasons we will define a framed tangle as a tangle: (a) with boundary lying on two lines, the upper one $\mathbb{R} \times\{0\} \times\{1\}$ and the lower one $\mathbb{R} \times\{0\} \times\{0\}$, and (b) equipped with a non-zero normal vector field which is standard $(0,1,0)$ at every boundary point. Framed tangles are considered up to isotopy as usual. In $\mathbb{R}^{3}$ the set of framing of each component can be canonically identified with $\mathbb{Z}$. The framed Kontsevich integral of a framed tangle $L$ is denoted by $Z(L)$. (For details, see $[3,4,19,20]$. In $[19,20], Z(L)$ is denoted by $\hat{Z}_{f}(L)$.)

If a framed tangle $L^{\prime}$ is obtained from another $L$ by increasing the framing of a component labeled $x$ by 1 , then we have the following framing formula:

$$
Z\left(L^{\prime}\right)=Z(L) \# \exp \left(\frac{1}{2} \bumpeq\right) .
$$

where the connected sum is done on the component labeled $x$ and $\_\in \mathcal{A}(\uparrow) \equiv$ $\mathcal{A}(\circlearrowright)$ is the Jacobi diagram based on $\circlearrowright$ with one strut.

The framed Kontsevich integral depends on the positions of the boundary points. To get rid of this dependence one has to choose standard positions for the boundary points. It turns out that the best "positions" are in a limit, when all the boundary points go to one fixed point. (One has to regularize the Kontsevich integral in the limit.) In the limit one has to keep track of the order in which the boundary points go to the fixed point. This leads to the notion of parenthesized framed tangle, or q-tangle in [19], - a framed tangle with a non-associative structure on each of the two sequences of boundary points on the upper and lower lines. For details, see [4, 19].

In all framed tangles in this paper, we assume that a non-associative structure is fixed. In many cases, there is only one non-associative structure, or the non-associative structure is clear from the context.

\subsection{Coproduct and Sliding property}

Let

$$
\begin{aligned}
\Delta_{x_{1} \ldots x_{n}}^{x}: \mathcal{A}\left(\uparrow_{x} \cup X\right) & \rightarrow \mathcal{A}\left(\uparrow_{x_{1}} \cup \cdots \cup \uparrow_{x_{n}} \cup X\right) \\
\Delta_{x_{1} \ldots x_{n}}^{x}: \mathcal{A}\left(\circlearrowright_{x} \cup X\right) & \rightarrow \mathcal{A}\left(\circlearrowright_{x_{1}} \cup \cdots \cup \circlearrowright_{x_{n}} \cup X\right)
\end{aligned}
$$

be the pull-back of the $n$-fold disconnected cover of the component labeled $x$. When we do not care about the labels on the result, an alternate notation is $\Delta_{x}^{(n)}$. 
Suppose $D \in \mathcal{A}\left(\uparrow_{x_{1}} \cup \cdots \cup \uparrow_{x_{n}}\right)$ and $D^{\prime} \in \mathcal{A}\left(\uparrow_{x_{1}} \cup \cdots \cup \uparrow_{x_{n}} \cup X\right)$. We define $D \cdot D^{\prime} \in \mathcal{A}\left(\uparrow_{x_{1}} \cup \cdots \cup \uparrow_{x_{n}} \cup X\right)$ as the element obtained by placing $D$ on top of $D^{\prime}$, ie, identifying the lower endpoint of $\uparrow_{x_{i}}$ in $D$ with the upper endpoint of $\uparrow_{x_{i}}$ in $D^{\prime}$, for $i=1, \ldots, n$. Similarly, $D^{\prime} \cdot D \in \mathcal{A}\left(\uparrow_{x_{1}} \cup \cdots \cup \uparrow_{x_{n}} \cup X\right)$ is obtained by placing $D^{\prime}$ on top of $D$.

In general $D \cdot D^{\prime} \neq D^{\prime} \cdot D$. The following is a special case when one has equality (see, for example, [20, Lemma 8.1]):

Lemma 3.1 (Sliding property) The image of $\Delta_{x}^{(n)}$ commutes with $\mathcal{A}\left(\uparrow_{x_{1}}\right.$ $\left.\cup \cdots \cup \uparrow_{x_{n}}\right)$, ie, for every $D \in \mathcal{A}\left(\uparrow_{x_{1}} \cup \cdots \cup \uparrow_{x_{n}}\right)$ and $D^{\prime} \in \mathcal{A}\left(\uparrow_{x} \cup X\right)$, we have that $D \cdot \Delta_{x}^{(n)}\left(D^{\prime}\right)=\Delta_{x}^{(n)}\left(D^{\prime}\right) \cdot D$.

With the above product, $\mathcal{A}\left(\uparrow_{x_{1}} \cup \cdots \cup \uparrow_{x_{n}}\right)$ is an algebra. There is also a coproduct on $\mathcal{A}\left(\uparrow_{x_{1}} \cup \cdots \cup \uparrow_{x_{n}}\right)$ which gives us a structure of a Hopf algebra, and $\mathcal{A}\left(\uparrow_{x_{1}} \cup \cdots \cup \uparrow_{x_{n}}\right)$ is a (completed) polynomial algebra generated by primitive elements. The isolated chord diagrams are among primitive elements. This is the reason why there is a canonical algebra embedding from $\mathcal{A}^{\prime}\left(\uparrow_{x_{1}} \cup \cdots \cup \uparrow_{x_{n}}\right)$ into $\mathcal{A}\left(\uparrow_{x_{1}} \cup \cdots \cup \uparrow_{x_{n}}\right)$.

\subsection{Disconnected cabling}

Suppose $L$ is a framed tangle, with one of its components labeled $x$. The $n$-fold disconnected cabling of $L$ along $x$, denoted by $\Delta_{x}^{(n)}(L)$, is the tangle obtained from $L$ by replacing the component labeled $x$ with $n$ of its parallels. Here the parallels are determined by the framing, and each inherits a natural framing from that of component $x$.

The following proposition, proved in [20], describes the behaviour of the Kontsevich integral under disconnected cabling.

Proposition 3.2 Suppose that a component labeled $x$ in a framed tangle $L$ is either closed or has one upper and one lower boundary points. Then

$$
Z\left(\Delta_{x}^{(n)} L\right)=\Delta_{x}^{(n)}(Z(L)) .
$$

Since $Z\left(\Delta_{x}^{(n)} L\right)$ depends on the positions of the boundary points, one has to be careful about the boundary points of the new components (ie parallels) in $\Delta_{x}^{(n)} L$ when the components label $x$ is not closed. The correct choice is the one in which the distances between the boundary points of the parallels are 
infinitesimally small compared to the distance between any of these points and any other boundary point. In the language of parenthesized framed tangles (or $q$-tangles), this means the boundary points of the parallels must form an innermost structure in the overall non-associative structure of the tangle $L^{\prime}$, and the non-associative structure among the boundary points of the parallels on the upper line must be the same as that among the boundary points of the parallels on the lower line.

Remark 3.3 In general, the disconnected cabling formula (6) does not hold true if the $x$ component has both boundary points on the same upper or lower line. However, it would hold true if the framed Kontsevich integral is modified by using a good enough associator [20].

\subsection{Connected cabling}

Let us define

$$
\psi_{x}^{(n)}: \mathcal{A}\left(\circlearrowright_{x} \cup X\right) \rightarrow \mathcal{A}\left(\circlearrowright_{x} \cup X\right)
$$

as the pull-back of the $n$-fold connected cover of the circle labeled $x$.

Suppose $L$ is a framed tangle, with one of its closed components labeled by $x$. The $n$-fold connected cabling of $L$ along $x$, denoted by $\mathrm{C} \Delta_{x}^{(n)}(L)$, is defined as follows. On the torus boundary of a small tubular neighborhood of component $x$ there are the preferred longitude and meridian. Replace the component $x$ with a closed curve on the torus boundary whose homology class is equal to that of the meridian plus $n$ times the longitude. The result is $\mathrm{C} \Delta_{x}^{(n)}(L)$. The new component inherits the orientation and framing from the old one.

The following theorem describes the behaviour of the Kontsevich integral under connected cabling.

Theorem 1 Suppose a component labeled $x$ in a framed tangle $L$ is closed (ie a knot). Then

$$
Z\left(\mathrm{C} \Delta_{x}^{(n)}(L)\right)=\left[\psi_{x}^{(n)}\left(Z(L) \#_{x} \exp \left(\frac{1}{2 n} \_\right)\right)\right] \# \exp \left(-\frac{1}{2}\llcorner) .\right.
$$

Proof We will prove the theorem in the case when $L$ is a knot. The case of an arbitrary tangle is quite similar.

The difference between the connected cabling and the disconnected cabling is the extra $1 / n$ twist $T_{n}$ inserted at one point:

$$
T_{n}=\frac{11 Y}{111} \text {. }
$$


By isotopy we can assume that this twist occurs in a horizontal slice where all the other strands are vertical. We can apply $(6)$ on the $(n, n)$ "tangle" obtained by excising $T_{n}$. (This object is not properly a tangle, since there is a little piece cut out of it. But we can still compute its Kontsevich integral.) To complete the computation, we need to compute $a:=Z\left(T_{n}\right)$.

Repeating $T_{n} n$ times, we get a full twist which we can compute using the framing and the disconnected cabling formulas (5), (6):

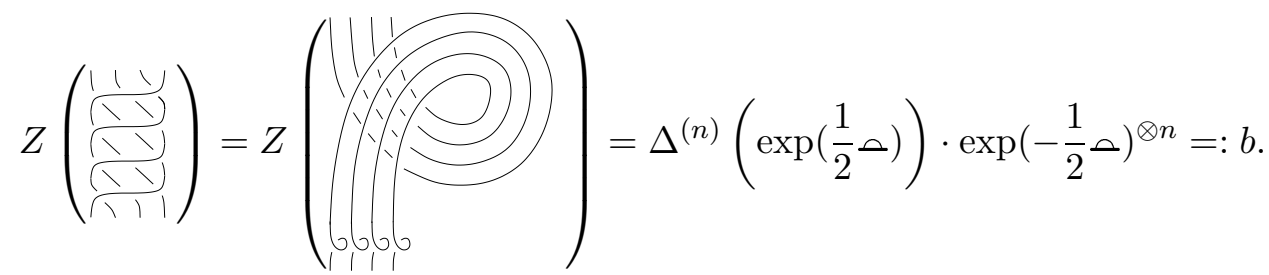

The notation $\left.\exp \left(-\frac{1}{2}\right\lrcorner\right)^{\otimes n}$ means $n$ copies of the framing change element $\left.\exp \left(-\frac{1}{2}\right\lrcorner\right)$, one on on each of the $n$ strands, and the product $\cdot$ is the product in $\mathcal{A}(\uparrow \cup \cdots \cup \uparrow)$.

The $n$ copies of $T_{n}$ that appear are not quite the same: they differ by cyclic permutations of the strands. If we could arrange the $n$ strands at the top and bottom of $T_{n}$ to be at the vertices of a regular $n$-gon, the strands would be symmetric and $a^{n}=b$ or

$$
a=b^{\frac{1}{n}}=\Delta^{(n)}\left(\exp \left(\frac{1}{2 n} \_\right)\right) \exp \left(-\frac{1}{2 n} \_\right)^{\otimes n} .
$$

In reality, $a$ is not symmetric, ie, $\sigma(a) \neq a$, where $\sigma$ is the automorphism of $\mathcal{A}\left(\uparrow_{x_{1}} \ldots \uparrow_{x_{n}}\right)$ which rotates the strands by $x_{i} \mapsto x_{i-1}$. We have

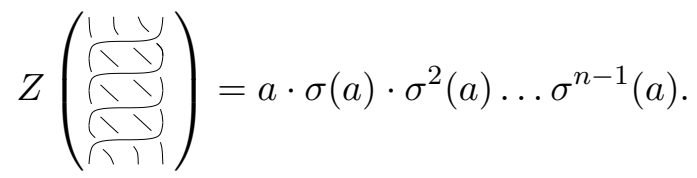

We can conjugate $T_{n}$ by some tangle $C$ to get the strands symmetric: $T_{n}=$ $C T_{n}^{\prime} C^{-1}$, with $T_{n}^{\prime}$ symmetric. From the definition of the framed Kontsevich integral [19], it follows that $a=c \cdot a^{\prime} \cdot \sigma\left(c^{-1}\right)$, where $a^{\prime}=Z^{\prime}\left(T_{n}^{\prime}\right)$ is the usual Kontsevich integral of $T_{n}^{\prime}$, and $c=Z(C) \in \mathcal{A}\left(\uparrow_{x_{1}} \cup \cdots \cup \uparrow_{x_{n}}\right)$. Thus

$$
a \cdot \sigma(a) \cdot \sigma^{2}(a) \ldots \sigma^{n-1}(a)=c\left(a^{\prime}\right)^{n} c^{-1} .
$$

And hence

$$
\begin{aligned}
a & =c \cdot b^{\frac{1}{n}} \cdot c^{-1} \\
& =c \cdot \Delta^{(n)}\left(\exp \left(\frac{1}{2 n} \_\right)\right) \cdot \exp \left(-\frac{1}{2 n} \_\right)^{\otimes n} \cdot c^{-1} .
\end{aligned}
$$

Geometry $\&$ Topology, Volume 7 (2003) 
By the above computations, the invariant of the connected cable of a knot $L$ is $\Delta^{(n)}(Z(L))$, multiplied by $a=Z\left(T_{n}\right)$, and close up with a twist. The conjugating elements $c$ and $c^{-1}$ can be swept through the knot, using the sliding property of Lemma 3.1, and cancel each other. The factor $\Delta^{(n)}(\exp (\llcorner/ 2 n))$ in $a$ can be combined with $Z(L)$ so that we apply $\Delta^{(n)}$ to $Z(L) \# \exp \left(\_/ 2 n\right)$. The twisted closure turns $\Delta^{(n)}$ into $\psi^{(n)}$. The remaining $n$ factors of $\exp \left(-\_/ 2 n\right)$ in $a$ can be slid around the knot and combined to give

$$
Z\left(\mathrm{C} \Delta^{n}(L)\right)=\left[\psi^{(n)}\left(Z(L) \# \exp \left(\frac{1}{2 n} \bullet\right)\right)\right] \# \exp \left(-\frac{1}{2} \bullet\right) .
$$

Remark 3.4 Suppose $\mathrm{C} \Delta_{x}^{(n \mid m)}(L)$ is the connected $(n, m)$-cabling of a framed tangle $L$ along a closed component labeled $x$, where $n$ and $m$ are co-prime integer with $n>0$, ie, $\mathrm{C} \Delta_{x}^{(n \mid m)}(L)$ is obtained by replacing the $x$ component with a closed curve on the torus boundary of the regular neighborhood which represents the homology class of $m$ times the meridian plus $n$ times the longitude. Let $\psi^{(n \mid m)}$ denotes the corresponding pull-back of Jacobi diagrams. Then the proof of Theorem 1 also gives:

$$
\left.\left.Z\left(\mathrm{C} \Delta^{(n \mid m)}(L)\right)=\left[\psi^{(n \mid m)}\left(Z(L) \# \exp \left(\frac{m}{2 n}\right\lrcorner\right)\right)\right] \# \exp \left(-\frac{m}{2}\right\lrcorner\right) .
$$

\subsection{Operators $\Delta, \psi$ and symmetrized diagrams}

One reason to introduce symmetrized diagrams is that the operations $\Delta$ and $\psi$ above become very simple in $\mathcal{B}$. Using the symmetrization map one can trade an interval in the skeleton with an asterisk, and a circle with a circled asterisk. The map $\Delta_{x}$ and $\psi_{x}$ can be carried over to the new spaces. The following lemmas are well-known (and easy to check).

Lemma 3.5 The map

$$
\Delta_{x_{1} \ldots x_{n}}^{x}: \mathcal{A}\left(*_{x} \cup X\right) \rightarrow \mathcal{A}\left(*_{x_{1}} \cup \cdots \cup *_{x_{n}} \cup X\right)
$$

is the sum over all ways of replacing each $x$ leg by one of the $x_{i}$.

Remark 3.6 $\Delta$ is similar to a coassociative, cocommutative coproduct in a coalgebra, except that it does not take values in $\mathcal{A} \otimes \mathcal{A}$.

The operation $\Delta$ in Lemma 3.5 is analogous to a change of variables $x \mapsto$ $x_{1}+\cdots+x_{n}$ for ordinary functions $f(x)$. We will use a suggestive notation: a leg labeled by a linear combination of variables means the sum over all ways 
of picking a variable from the linear combination. If $D(x)$ is a diagram with some legs labeled $x, \Delta^{(n)}(D(x))=D\left(x_{1}+\cdots+x_{n}\right)$ is the diagram with the same legs labeled $x_{1}+\cdots+x_{n}$.

Lemma 3.7 (See [17]) The map

$$
\psi_{x}^{(n)}: \mathcal{A}\left(\circledast_{x} \cup X\right) \rightarrow \mathcal{A}\left(\circledast_{x} \cup X\right)
$$

is multiplication by $n^{k}$ on diagrams with $k$ legs labeled $x$.

This operation is related to the change of variables $x \mapsto n x$.

\section{The Wheeling Theorem}

The operator $\partial_{\Omega}: \mathcal{B} \rightarrow \mathcal{B}$, where $\Omega$ is the wheels element of the Introduction, is called the "wheeling" map. The proof of the following theorem will occupy the rest of this section.

Theorem 2 (Wheeling) The map $\Upsilon=\chi \circ \partial_{\Omega}: \mathcal{B} \rightarrow \mathcal{A}$ is an algebra isomorphism.

The map $\Upsilon$ is the diagrammatic analogue of the Duflo-Kirillov map. Note that by (4), $\partial_{\Omega} \partial_{\Omega^{-1}}=\mathrm{id}$, hence $\partial_{\Omega}$ is a vector space isomorphism. Since $\chi$ is also a vector space isomorphism, $\Upsilon$ is automatically bijective.

\subsection{An inner product}

Suppose $C, C^{\prime} \in \mathcal{B}$ are diagrams such that $C$ has no struts. If $C$ and $C^{\prime}$ have the same number of legs, then the inner product $\left\langle C, C^{\prime}\right\rangle$ is the sum of all ways of gluing all the legs of $C$ to all legs of $C^{\prime}$. If $C$ and $C^{\prime}$ do not have the same number of legs, then define $\left\langle C, C^{\prime}\right\rangle=0$. The restriction that $C$ not have struts is to guarantee convergence and avoid closed circles.

We will sometimes want to fix $C$ and consider $\langle C, \cdot\rangle$ as a map from $\mathcal{B}$ to $\mathcal{A}(\emptyset)$; we will denote this map $\iota(C)$. This definition works equally well in the presence of other skeleton components or to glue several components. We will use subscripts to indicate which ends are glued.

There are two dualities relating $\langle\cdot, \cdot\rangle$ with other operations we have defined. 
Lemma 4.1 Multiplication and comultiplication in $\mathcal{B}$ are dual in the sense that

$$
\left\langle C, D_{1} \sqcup D_{2}\right\rangle=\left\langle\Delta_{x y} C,\left(D_{1}\right)_{x} \otimes\left(D_{2}\right)_{y}\right\rangle_{x y} .
$$

Similar statements hold in the presence of other ends.

Proof The glued diagrams are the same on the two sides; we either combine the legs of $D_{1}$ and $D_{2}$ into one set and then glue with $C$, or we split the legs of $C$ into two pieces which are then glued with $D_{1}$ and $D_{2}$. (Note that there are no combinatorial factors to worry about: in both cases, we take the sum over all possibilities.)

Lemma 4.2 Multiplication by a diagram $B \in \mathcal{B}$ and applying $B$ as a diagrammatic differential operator are adjoint in the sense that

$$
\langle A \sqcup B, C\rangle=\left\langle A, \partial_{B}(C)\right\rangle .
$$

Proof As before, the diagrams are the same on both sides.

\subsection{The map $\Phi$}

Let $\psi_{x}^{z}$ be the tangle in Figure 1(a), which is a bead (labeled $x$ here) on a wire (labeled $z$ here). Its Kontsevich integral $Z\left({ }_{x}^{z}\right)$ takes values in $\mathcal{A}\left(\uparrow_{z}, \circlearrowright_{x}\right)$. Symmetrizing the legs attached to the bead $x$ as explained in 2.4, we get

$$
\chi_{x}^{-1} Z\left(\varphi_{x}^{z}\right) \in \mathcal{A}\left(\uparrow_{z}, \circledast_{x}\right) .
$$

Finally, we use the inner product operation along the legs $x$ to get a map from $\mathcal{B}$ to $\mathcal{A}$ :

$$
\Phi=\iota_{x} \chi_{x}^{-1} Z\left(\oplus_{x}^{z}\right): \mathcal{B} \rightarrow \mathcal{A}
$$

In this last step, there are two things we have to check. First, we must see that $\chi^{-1} Z(\phi)$ has no struts. This follows from the fact that we took the bead with the zero framing. Second, we need to check that the inner product descends modulo the link relations on $x$ in $\mathcal{A}\left(\uparrow_{z}, \circledast_{x}\right)$.

Lemma 4.3 The inner product $\langle\cdot, \cdot\rangle_{x}: \mathcal{A}\left(*_{x} \cup X\right) \otimes \mathcal{A}\left(*_{x}\right) \rightarrow \mathcal{A}(X)$ descends to a map $\langle\cdot, \cdot\rangle_{x}: \mathcal{A}\left(\circledast_{x} \cup X\right) \otimes \mathcal{A}\left(*_{x}\right) \rightarrow \mathcal{A}(X)$.

Proof Link relations in $\mathcal{A}\left(\circledast_{x} \sqcup X\right)$ can be slid over diagrams in $\mathcal{A}\left(*_{x}\right)$, as shown in Figure 4. (See similar arguments in [3]). 


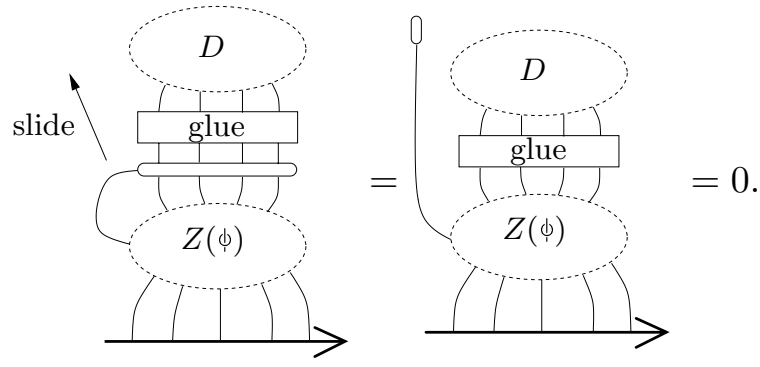

Figure 4: The proof that $\Phi(D)$ is well-defined modulo link relations on $Z(\downarrow)$ : link relations in $Z(\underset{1}{)})$ can be slid over $D$.

\subsection{Multiplicativity of $\Phi$}

We now come to the key lemma in the proof of the wheeling theorem.

Lemma 4.4 The map $\Phi: \mathcal{B} \rightarrow \mathcal{A}$ is an algebra map.

Proof As advertised, we use the equality of links " $1+1=2$ ". Let us see what this equality of links says about the Kontsevich integral of the Hopf link. On the " $1+1$ " side, we see the connected sum of two open Hopf links. It is known that the invariant of the connected sum is the connected sum of the invariants. To write this conveniently, let $H(z ; x)$ be $Z\left(\phi_{1}\right) \in \mathcal{A}\left(\uparrow_{z}, \circledast x\right)$, with the wire labeled by $z$ and the bead labeled by $x$. Then

$$
Z(\downarrow \# \downarrow)=H\left(z ; x_{1}\right) \#_{z} H\left(z ; x_{2}\right) \in \mathcal{A}\left(\uparrow_{z}, \circledast_{x_{1}}, \circledast_{x_{2}}\right) .
$$

On the "2" side, we see the disconnected cable of a Hopf link. By the disconnected cabling formula (6), this becomes the coproduct $\Delta$ :

$$
Z\left(\Delta_{x}^{(2)}\left(\biguplus_{1}\right)=\Delta_{x_{1} x_{2}}^{x} H(z ; x) \in \mathcal{A}\left(\uparrow_{z}, \circledast_{x_{1}}, \circledast_{x_{2}}\right) .\right.
$$

Since the two tangles are isotopic, we have

$$
H\left(z ; x_{1}, x_{2}\right) \stackrel{\text { def }}{=} H\left(z ; x_{1}\right) \#_{z} H\left(z ; x_{2}\right)=\Delta_{x_{1} x_{2}}^{x} H(z ; x) \in \mathcal{A}\left(\uparrow_{z}, \circledast_{x_{1}}, \circledast_{x_{2}}\right) .
$$

Now consider the map

$$
\Xi=\iota_{x_{1}} \iota_{x_{2}} H\left(z ; x_{1}, x_{2}\right): \mathcal{B} \otimes \mathcal{B} \rightarrow \mathcal{A} ;
$$

in other words, in $\Xi\left(D_{1} \otimes D_{2}\right)$ glue the $x_{1}$ and $x_{2}$ legs of $H\left(z ; x_{1}, x_{2}\right)$ to $D_{1}$ and $D_{2}$ respectively. This descends modulo the two different link relations in $\mathcal{A}(\uparrow, \circledast, \circledast)$ by the argument of Figure 4 , applied to $D_{1}$ and $D_{2}$ separately. We have two different expressions for this map from the two different expressions 
for $H\left(z ; x_{1}, x_{2}\right)$. On the " $1+1$ " side, the gluing does not interact with the connected sum and we have

$$
\Xi\left(D_{1}, D_{2}\right)=\Phi\left(D_{1}\right) \# \Phi\left(D_{2}\right),
$$

see Figure 5.

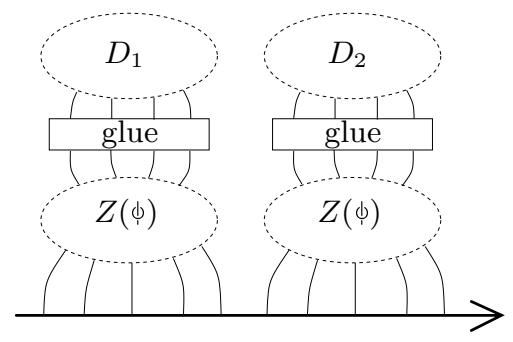

Figure 5: Gluing $Z(\downarrow \#$

For the "2" side, we use Lemma 4.1 to see that

$$
\begin{aligned}
\Xi\left(D_{1}, D_{2}\right) & =\left\langle\Delta^{x} H(z ; x), D_{1} \otimes D_{2}\right\rangle \\
& =\left\langle H(z ; x), D_{1} \sqcup D_{2}\right\rangle \\
& =\Phi\left(D_{1} \sqcup D_{2}\right),
\end{aligned}
$$

see Figure 6.

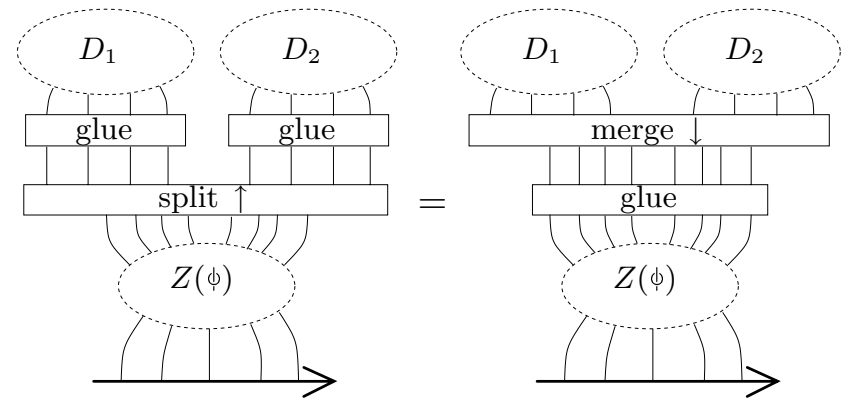

Figure 6: Gluing $Z\left(\Delta_{x}^{(2)}\left(\biguplus_{1}\right)\right)$ to $D_{1} \otimes D_{2}$ in two equivalent ways

Combining the two, we find

$$
\Phi\left(D_{1}\right) \# \Phi\left(D_{2}\right)=\Phi\left(D_{1} \sqcup D_{2}\right) .
$$

Geometry $8 \mathcal{T}$ Topology, Volume 7 (2003) 


\subsection{Mapping degrees and the Duflo-Kirillov isomorphism}

We have successfully constructed a multiplicative map from $\mathcal{B}$ to $\mathcal{A}$. We will see later that this map $\Phi$ is the same as $\Upsilon$, but we cannot yet see this. Instead we will consider the lowest degree term $\Phi_{0}$ of $\Phi$.

The mapping degree of a diagram $D \in \mathcal{A}\left(\uparrow_{z}, \circledast_{x}\right)$ with respect to $x$ is the amount $\iota_{x} D: \mathcal{B} \rightarrow \mathcal{A}$ shifts the degree. Explicitly, it is the degree of $D$ minus the number of $x$ legs of $D$.

Since there are no $x-x$ struts in $H(z ; x)$, every $x$ leg of $H$ must be attached to another vertex (either internal or on the interval $z$ ). Furthermore, if two $x$ legs are attached to the same internal vertex, the diagram vanishes by antisymmetry. Therefore there are at least as many other vertices as $x$ legs in $H$ and the mapping degree is $\geq 0$.

Let $H_{0}(z ; x)$ be the part of $H(z ; x)$ of mapping degree 0 with respect to $x$, and $\Phi_{0}: \mathcal{B} \rightarrow \mathcal{A}$ be $\iota_{x} H_{0}(z ; x)$. The map $\Phi_{0}$ is still multiplicative, since the multiplications in $\mathcal{A}$ and $\mathcal{B}$ both preserve degrees. (For homogeneous diagrams $D_{1}$ and $D_{2}$ of degrees $n_{1}$ and $n_{2}, \Phi_{0}\left(D_{1} \sqcup D_{2}\right)$ is the piece of $\Phi\left(D_{1} \sqcup D_{2}\right)$ of degree $n_{1}+n_{2}$ and likewise for $\Phi_{0}\left(D_{1}\right) \# \Phi_{0}\left(D_{2}\right)$.)

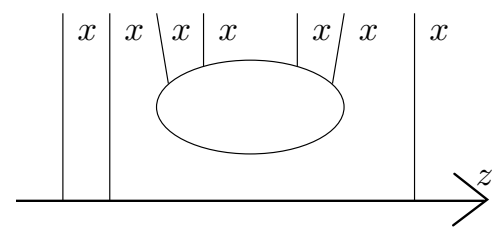

Figure 7: The only diagrams in $\mathcal{A}\left(\uparrow_{z}, \circledast_{x}\right)$ of mapping degree 0 with respect to $x$ are wheels and struts.

The diagrams that appear in $H_{0}$ are very restricted, since every vertex that is not an $x$ leg must connect to an $x$ leg. The possible diagrams are $x$ wheels and $x-z$ struts, as shown in Figure 7. The linking number between the bead and the wire in the link $\downarrow$ is 1 , so the coefficient of the $x-z$ strut is 1 . Combined with the fact that the Kontsevich integral is grouplike [20], we find that

$$
\begin{aligned}
H_{0}(z ; x) & =\underset{z}{x}(\mid) \sqcup \Omega^{\prime}, \quad \text { where } \\
\Omega^{\prime} & =\exp _{\sqcup}\left(\sum_{n} a_{2 n} \omega_{2 n}\right)
\end{aligned}
$$

for some coefficients $a_{2 n}$. Note that the right hand side is written in $\mathcal{A}(\uparrow, *)$ (with a strange mixed product), since there is no algebra structure on $\mathcal{A}(\uparrow, \circledast)$. 
By the following lemma, we now have a multiplicative map very similar to our desired map $\Upsilon$.

Lemma 4.5 One has that $\Phi_{0}=\chi \circ \partial_{\Omega^{\prime}}$.

Proof Using Lemma 4.2 and noting that gluing with $\exp \left(\left.\right|_{z} ^{x}\right)$ takes the legs of a diagram in $\mathcal{B}$ and averages over all ways of ordering them, as in the definition of $\chi$, we see that

$$
\Phi_{0}(D)=\left\langle\underset { z } { \operatorname { e x p } } \left(\left|\sqcup \Omega^{\prime}, D\right\rangle=\left\langle\underset { z } { \operatorname { e x p } } \left(\left|, \partial_{\Omega}^{\prime}(D)\right\rangle=\chi\left(\partial_{\Omega}^{\prime}(D)\right) .\right.\right.\right.\right.
$$

\subsection{Identifying $\Phi_{0}$ with $\Upsilon$}

To complete the proof of the wheeling theorem, one needs only to show that $\Omega=\Omega^{\prime}$, or $a_{n}=b_{n}$ for $n=2,4,6, \ldots$ This can be proved as follows.

First of all, a calculation of the degree 2 part of the Kontsevich integral of the Hopf link will show that $a_{2}=b_{2}$. Thus if $\Omega \neq \Omega^{\prime}$, then for some $n>1$,

$$
\Omega^{-1} \Omega^{\prime}=1+\left(a_{2 n}-b_{2 n}\right) \omega_{2 n}+\text { higher order terms. }
$$

Second, the map $\Upsilon=\chi \circ \partial_{\Omega}$ is known to be an algebra isomorphism on the level of simple Lie algebras [5]. Thus for a simple Lie algebra $\mathfrak{g}$, the map $\partial_{\Omega^{-1} \Omega^{\prime}}$ is an algebra automorphism of $S(\mathfrak{g})^{\mathfrak{g}}$. When $\mathfrak{g}=s l_{2}$, the algebra $S(\mathfrak{g})^{\mathfrak{g}}$ is a polynomial algebra on one generator, which is the image of the strut $\frown$. On the strut $\partial_{\Omega^{-1} \Omega^{\prime}}$ acts as the identity (since there is no non-trivial diagram with less than 3 legs), hence $\partial_{\Omega^{-1} \Omega^{\prime}}$ acts as the identity on the whole algebra $S(\mathfrak{g})^{\mathfrak{g}}$.

Third, the action of $\omega_{2 n}$ on $S(\mathfrak{g})^{\mathfrak{g}}$ is non-trivial. Explicitly, $\partial_{\omega_{2 n}}\left[(\frown)^{n}\right]=$ $2(2 n+1)$ ! in $s l_{2}$, which can be proved easily by induction. Thus, if $a_{2 n} \neq b_{2 n}$, then $\partial_{\Omega^{-1} \Omega^{\prime}}$ cannot act as identity on $S(\mathfrak{g})^{\mathfrak{g}}$.

We conclude that $\Omega=\Omega^{\prime}$, and this completes the proof of the wheeling theorem. For another proof of $\Omega=\Omega^{\prime}$, more detailed and without using the result of [5], see the Appendix.

\subsection{Back to the Duflo-Kirillov isomorphism}

We note that the wheeling theorem implies the multiplicative property of the Duflo-Kirillov isomorphism for a metrized Lie (super-) algebra $\mathfrak{g}$. Indeed, using the standard maps $W_{\mathfrak{g}}$ from spaces of diagrams into spaces of tensors, we set 
$J=W_{\mathfrak{g}}(H(z ; x)) \in U(\mathfrak{g}) \otimes S(\mathfrak{g})_{\mathfrak{g}}$. Here $S(\mathfrak{g})_{\mathfrak{g}}$ denotes the space of coinvariants of the $\mathfrak{g}$ action on $S(\mathfrak{g})$ - the link relation dictates the descent to this quotient of $S(\mathfrak{g})$. Also, strictly speaking $J$ lives in the completion of $U(\mathfrak{g}) \otimes S(\mathfrak{g})_{\mathfrak{g}}$ induced by the grading on $S(\mathfrak{g})_{\mathfrak{g}}$. Equation $(7)$ and the compatibility between $W_{\mathfrak{g}}$ and multiplication and comultiplication imply now that $J$ satisfies

$$
J \# J=(1 \otimes \Delta) J \quad \text { in } \quad U(\mathfrak{g}) \otimes S(\mathfrak{g})_{\mathfrak{g}} \otimes S(\mathfrak{g})_{\mathfrak{g}},
$$

where $J \# J$ denotes the result of multiplying two copies of $J$ using the product of $U(\mathfrak{g})$, so that the result is in (the appropriate completion of) $U(\mathfrak{g}) \otimes S(\mathfrak{g})_{\mathfrak{g}} \otimes$ $S(\mathfrak{g})_{\mathfrak{g}}$. Now use the metric of $\mathfrak{g}$ to identify the space of coinvariants in $S(\mathfrak{g})$ as the dual of the space $S(\mathfrak{g})^{\mathfrak{g}}$ of invariants and hence to re-interpret $J$ as an element of $U(\mathfrak{g}) \otimes\left(S(\mathfrak{g})^{\mathfrak{g}}\right)^{\star}$ and hence as a map $W_{J}: S(\mathfrak{g})^{\mathfrak{g}} \rightarrow U(\mathfrak{g})$. One easily verifies that equation (11) implies that $W_{J}$ is multiplicative. It remains to see that $W_{J}$ is equal to the Duflo-Kirillov map $\chi \circ \partial_{j^{\frac{1}{2}}}$. This follows from the computation of $H(z ; x)$ in terms of the diagrammatic analogue $\Omega$ of $j^{\frac{1}{2}}$ in Section 6 .

\section{The Wheels Theorem. The Kontsevich integral of the unknot}

This section is devoted to the proof of the Wheels theorem.

Theorem 3 (Wheels) The framed Kontsevich integral of the unknot is the wheels element:

$$
Z(\circlearrowright)=\chi(\Omega) .
$$

We will denote $\nu=Z(\circlearrowright) \in \mathcal{A}=\mathcal{A}(\uparrow) \equiv \mathcal{A}(\circlearrowright)$.

\subsection{Useful facts}

We will first derive some nice properties the wheels element $\Omega$. Set $H_{0}(z ; x)=$ $\Omega_{x} \exp \left({ }^{x} \frown^{z}\right)$ and start from the basic equality proved in the Wheeling theorem,

$$
\Delta_{x_{1} x_{2}}^{x} H_{0}(z ; x)=H_{0}\left(z ; x_{1}\right) \#_{z} H_{0}\left(z ; x_{2}\right) \in \mathcal{A}\left(\uparrow_{z} \circledast_{x_{1}} \circledast_{x_{2}}\right) .
$$

Now consider dropping the strand $z$, ie, mapping all diagrams with a $z$ vertex to 0 . (Knot-theoretically, this corresponds to dropping the central strand in the equation " $1+1=2$ ".) We find

$$
\Delta \Omega=\Omega \otimes \Omega \in \mathcal{A}(\circledast \circledast) .
$$

Note that this equality is not true inside $\mathcal{A}(* *)$. 
Lemma 5.1 (Pseudo-linearity of $\log \Omega$, see also [7]) For any $D \in \mathcal{B}$,

$$
\partial_{D}(\Omega)=\langle D, \Omega\rangle \Omega \text {. }
$$

\section{Proof}

$$
\partial_{D}(\Omega)_{x}=\left\langle D_{y}, \Omega_{x+y}\right\rangle_{y}=\left\langle D_{y}, \Omega_{x} \Omega_{y}\right\rangle_{y}=\left\langle D_{y}, \Omega_{y}\right\rangle_{y} \Omega_{x} .
$$

In the second equality, we use Equation (12). This is allowed, since the contraction descends to $\mathcal{A}(\circledast \circledast) \simeq \mathcal{A}(\circledast \uparrow)$ by the argument of Lemma 4.3.

Remark 5.2 Compare this lemma with standard calculus: if $D$ is any differential operator and $f$ is a linear function, then $D e^{f}=(D f)(0) e^{f}$. The prefix "pseudo" is written above because Lemma 5.1 does not hold for every $D$, but only for $x$-invariant $D$ 's, ie, for $D$ with link relations on $x$-legs.

Although we are interested in knots and links in $S^{3}$, for which the appropriate space of diagrams is the boundary connected part $\mathcal{A}^{\text {bc }}$, vacuum diagrams (elements of $\mathcal{A}(\emptyset)$ ) appear at various points. Notably, the wheeling map $\Upsilon$ does not preserve the subspace of boundary connected diagrams. Although the resulting vacuum components can be computed explicitly, ${ }^{2}$ they are almost always irrelevant for us and it would just complicate the formulas to keep track of them. To avoid this, we will introduce the boundary-connected projection $\pi^{\mathrm{bc}}: \mathcal{A} \rightarrow \mathcal{A}^{\mathrm{bc}}$ which maps any diagram containing vacuum components to 0 and is otherwise the identity. Note that $\pi^{\mathrm{bc}}$ is multiplicative. There are similar projections, which will also be called $\pi^{\mathrm{bc}}$, for other spaces $\mathcal{A}(X)$.

If we compose Lemma 5.1 with $\pi^{\mathrm{bc}}$, we find, for a diagram $D \in \mathcal{B}$,

$$
\pi^{\mathrm{bc}} \partial_{D} \Omega= \begin{cases}\Omega & D \text { is the empty diagram } \\ 0 & \text { otherwise. }\end{cases}
$$

\subsection{A lemma on the bound of numbers of legs}

Lemma 5.3 For any elements $x_{1}, \ldots, x_{k} \in \mathcal{A}(\uparrow)$ with at least one leg on the interval $\uparrow, \chi^{-1}\left(x_{1} \# \cdots \# x_{k}\right) \in \mathcal{B}$ has at least $k$ legs.

Proof First note that any vacuum diagrams that appear in the $x_{i}$ 's pass through unchanged to the result; let us assume that there are none, so that

${ }^{2}$ D. Bar-Natan and R. Lawrence [7] have done these computations 
we can use the vacuum projection $\pi^{\mathrm{bc}}$ without changing the result. By the wheeling theorem,

$$
\pi^{\mathrm{bc}} \chi^{-1}\left(x_{1} \# \ldots \# x_{n}\right)=\pi^{\mathrm{bc}} \partial_{\Omega}\left(\Upsilon^{-1}\left(x_{1}\right) \sqcup \cdots \sqcup \Upsilon^{-1}\left(x_{k}\right)\right) .
$$

Let $y_{i}=\pi^{\mathrm{bc}} \Upsilon^{-1}\left(x_{i}\right)$. Each $y_{i}$ has at least one leg, since if the $\partial_{\Omega}^{-1}$ of $\Upsilon^{-1}=$ $\partial_{\Omega}^{-1} \chi^{-1}$ eats all the legs of $\chi^{-1} x_{i}$, it also creates a vacuum diagram which is killed by $\pi^{\mathrm{bc}}$. Then

$$
\pi^{\mathrm{bc}} \partial_{\Omega}\left(y_{1} \ldots y_{k}\right)=\pi^{\mathrm{bc}}\left\langle\Omega_{a}, \Delta_{a b}\left(y_{1} \ldots y_{k}\right)\right\rangle_{a} .
$$

Let $\Delta_{a b} y_{i}=\left(y_{i}\right)_{a}+z_{i}$; diagrams in $z_{1}$ have at least one $b$ leg. We see that

$$
\begin{aligned}
\pi^{\mathrm{bc}}\left\langle\Omega_{a},\left(y_{1}\right)_{a} \Delta_{a b}\left(y_{2} \ldots y_{n}\right)\right\rangle_{a} & =\pi^{\mathrm{bc}}\left\langle\left(\partial_{y_{1}} \Omega\right)_{a}, \Delta_{a b}\left(y_{2} \ldots y_{k}\right)\right\rangle_{a} & & \text { by Lemma } 4.2 \\
& =0 & & \text { by Equation } 13 .
\end{aligned}
$$

Therefore

$$
\begin{aligned}
\pi^{\mathrm{bc}} \partial_{\Omega}\left(y_{1} \ldots y_{k}\right) & =\pi^{\mathrm{bc}}\left(\left\langle\Omega_{a},\left(y_{1}\right)_{a} \Delta_{a b}\left(y_{2} \ldots y_{k}\right)\right\rangle_{a}+\left\langle\Omega_{a}, z_{1} \Delta_{a b}\left(y_{2} \ldots y_{k}\right)\right\rangle_{a}\right) \\
& =\pi^{\mathrm{bc}}\left\langle\Omega_{a}, z_{1} \Delta_{a b}\left(y_{2} \ldots y_{k}\right)\right\rangle_{a} \\
& =\cdots \\
& =\pi^{\mathrm{bc}}\left\langle\Omega_{a}, z_{1} \ldots z_{k}\right\rangle_{a} .
\end{aligned}
$$

Each $z_{i}$ has at least one leg labeled $b$, so the product has at least $k$ legs labeled $b$ which are the legs in the result.

\subsection{Coiling the unknot. Proof of the Wheels theorem}

The basic equation we will use to identify $\nu=Z(\circlearrowright)$ is " $n \cdot 0=0$ " from the introduction: the $n$-fold connected cable of the unknot is the unknot with a new framing. The connected cabling formula of Theorem 1 implies that

$$
\psi^{(n)}\left(\nu \# \exp _{\#}\left(\frac{1}{2 n} \_\right)\right)=\nu \# \exp _{\#}\left(\frac{n}{2} \_\right) .
$$

This equation is true for all $n \in \mathbb{Z}, n>0$. In each degree, each side is a Laurent polynomial in $n$ of bounded degree; therefore, the two sides are equal as Laurent polynomials. The RHS is a polynomial in $n$, so both sides are polynomials (ie, have no negative powers of $n$.) Let us evaluate both sides at $n=0$. On the RHS, we get just $\nu$. For the LHS, recall how $\psi^{(n)}$ acts in the space $\mathcal{B}$ : it multiplies a diagram with $k$ legs by $n^{k}$ (see Lemma 3.7).

Consider expanding the exponential $\exp _{\#}(-/ 2 n)$ in the LHS of Equation 14. In the term with $\left(\_\right)^{k}$, there is a factor of $1 / n^{k}$ from the coefficient $1 / 2 n$. On 
the other hand, by Lemma 5.3, the product has at least $k$ legs, or $k+1$ if there

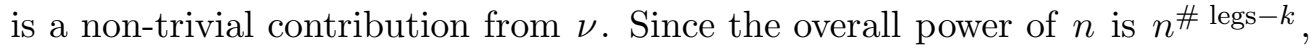
when we evaluate at $n=0$ the term $\nu$ does not contribute at all. Hence

$$
\left.\psi^{(n)}\left(\nu \# \exp _{\#}\left(\frac{1}{2 n} \_\right)\right)\right|_{n=0}=\left.\psi^{(n)}\left(\exp _{\#}\left(\frac{1}{2 n} \_\right)\right)\right|_{n=0} .
$$

Now we want to pick out the term from $\left(\llcorner)^{\# k}\right.$ with exactly $k$ legs. We can do this computation explicitly using the wheeling map $\Upsilon$. Alternatively, the result must be a diagram of degree $k$ and with $k$ legs, hence $\nu=\nu_{0}$, the part of mapping degree 0. It was shown in Section 4 that the part of of mapping degree 0 of $Z(\biguplus)$ is $\Omega_{x} \sqcup \exp _{\sqcup}\left(\left.\right|_{x} ^{z}\right)$. Dropping the central strand from $\downarrow$ leaves an unknot, so $\Omega=\nu_{0}=\nu$. This completes the proof of the Wheels theorem.

Exercise 5.4 Do the computation suggested above. Show that

$$
\left.\chi^{-1}\left(\exp _{\#}\left(\frac{1}{2}\right\lrcorner\right)\right)=\Omega \sqcup \exp _{\sqcup}\left(\frac{1}{2} \frown\right) .
$$

Hint 5.5 Use Lemma 6.3.

\section{From the unknot to the Hopf link}

By changing the framing on the unknot and cabling it, we can construct a Hopf link. Using the results of Section 3 and the value of $Z(\bigcirc)$, we can compute the invariant of the Hopf link from the invariant of the unknot. There are several good formulas for the answer. An alternative exposition of the results of this section can be found in [7].

Theorem 4 The framed Kontsevich integral of the Hopf link can be expressed in the following equivalent ways:

$$
\begin{aligned}
Z\left({ }_{x} \bigcirc_{y}\right) & =\left\{\begin{array}{l}
\Upsilon_{x} \circ \Upsilon_{y}\left(\exp \left({ }^{y} \frown^{x}\right)\right) \cdot(\text { Vacuum }) \\
\Upsilon_{x}\left(\exp _{\sqcup}\left(y \frown^{x}\right) \Omega_{x}\right) \cdot(\text { Vacuum })
\end{array}\right. \\
Z\left({ }_{x} y\right) & =\exp \left({ }^{y} \frown^{x}\right) \sqcup \Omega_{y},
\end{aligned}
$$

for some elements (Vacuum) $\in \mathcal{A}(\emptyset)$.

In the last expression, ${ }_{x}^{y}$ is the $(1,1)$ tangle whose closure is the Hopf link, with the bead labeled by $y$ and the wire labeled by $x$. From this last equality in Theorem 4, we can see exactly the map $\Phi$ from Section 4. 
Corollary 6.1 $\Phi=\Phi_{0}=\chi \circ \partial_{\Omega}$.

Proof (of Theorem 4) We start by computing the Kontsevich integral of the +1 framed unknot. In what follows we identify $\mathcal{B}$ and $\mathcal{A}$ using $\chi$, and use $\sqcup$ and \# to denote the two different products on $\mathcal{B}$.

$$
\begin{aligned}
Z\left(\bigcirc^{+1}\right) & \left.=\nu \# \exp _{\#}\left(\frac{1}{2}\right\lrcorner\right) & & \\
& =\partial_{\Omega}\left(\partial_{\Omega}^{-1}(\Omega) \sqcup \exp _{\sqcup}\left(\partial_{\Omega}^{-1}(\frown)\right)\right) & & \text { by Theorem } 2 \\
& =\pi^{\mathrm{bc}} \partial_{\Omega}(\Omega \sqcup \exp (\frown)) . & & \text { by Equation } 13
\end{aligned}
$$

To pass to the Hopf link, we double $Z\left(\bigcirc^{+1}\right)$. The following lemma, which is obvious from the definition, tells us how $\partial_{\Omega}$ interacts with doubling. We use $\hat{D}$ as an alternate notation for $\partial_{D}$ so that we can use a subscript to indicate which variable the differential operator acts on.

Lemma 6.2 For $C, D \in \mathcal{B}$ with $C$ strutless,

$$
\Delta_{x y} \hat{C}(D)=\hat{C}_{x}\left(\Delta_{x y} D\right)=\hat{C}_{y}\left(\Delta_{x y} D\right) .
$$

If we want to apply $\partial_{\Omega}^{-1}$ to both components of the Hopf link, we can compute $\partial_{\Omega}^{-2}\left(Z\left(\bigcirc^{+1}\right)\right)$.

Lemma 6.3 $\pi^{b c} \partial_{\Omega}\left(\exp \frac{1}{2} \frown\right)=\Omega \sqcup \exp \left(\frac{1}{2} \frown\right)$.

\section{Proof}

$$
\begin{array}{rlr}
\pi^{\mathrm{bc}} \partial_{\Omega}\left(\exp \left(\frac{1}{2} \frown\right)\right)=\pi^{\mathrm{bc}}\left\langle\Omega_{y}, \exp \left(\frac{1}{2} x+y \frown^{x+y}\right)\right\rangle_{y} & \\
& =\pi^{\mathrm{bc}}\left\langle\Omega_{y}, \exp \left(\frac{1}{2} \frown^{x}\right) \exp \left({ }^{y} \frown^{x}\right) \exp \left(\frac{1}{2} y \frown^{y}\right)\right\rangle_{y} & \\
& =\pi^{\mathrm{bc}}\left\langle\partial_{\exp \left(\frac{1}{2} \frown\right)}(\Omega)_{y}, \exp \left({ }^{x} \frown^{y}\right)\right\rangle_{y} \sqcup \exp \left(\frac{1}{2} \frown^{x} \frown^{x}\right) & \text { by Lemma } 4.2 \\
& =\pi^{\mathrm{bc}}\left\langle\Omega_{y}, \exp \left({ }^{y} \frown^{x}\right)\right\rangle_{y} \sqcup \exp \left(\frac{1}{2}{ }^{x} \frown^{x}\right) & \text { by Equation } 13 \\
& =\Omega \sqcup \exp \left(\frac{1}{2} \frown\right) .
\end{array}
$$

As a corollary, we see that

$$
\pi^{\mathrm{bc}} \partial_{\Omega}^{-2}\left(Z\left(\bigcirc^{+1}\right)\right)=\exp \left(\frac{1}{2} \frown\right) .
$$


We now compute.

$$
\begin{array}{rlrl}
\pi^{\mathrm{bc}} \Delta_{x y}\left(\hat{\Omega}^{-2} Z\left(\bigcirc^{+2}\right)\right)=\pi^{\mathrm{bc}} \hat{\Omega}_{x}^{-1} \hat{\Omega}_{y}^{-1} Z\left({ }_{x}^{+1} \bigodot_{y}^{+1}\right) & & \text { by Lemma } 6.2 \text { and } \\
= & \pi^{\mathrm{bc}} \Delta_{x y}\left(\exp \left(\frac{1}{2} \frown\right)\right) & & \text { by Equation } 15 \\
& =\exp \left({ }^{x} \frown^{y}\right) \exp \left(\frac{1}{2} \frown^{x}\right) \exp \left(\frac{1}{2} \frown^{y}\right) . &
\end{array}
$$

Apply $\Upsilon_{x} \circ \Upsilon_{y}$ to both sides. We see that

$$
\begin{aligned}
Z\left({ }_{x}^{+1} \bigcirc_{y}^{+1}\right) & =\pi^{\mathrm{bc}} Z\left(_{x}^{+1} \bigodot_{y}^{+1}\right) \\
& =\pi^{\mathrm{bc}} \Upsilon_{x} \circ \Upsilon_{y}\left(\exp \left({ }^{x} \frown^{y}\right) \sqcup \exp \left(\frac{1}{2} x \frown^{x}\right) \sqcup \exp \left(\frac{1}{2} y \frown^{y}\right)\right) \\
& \left.\left.=\pi^{\mathrm{bc}} \Upsilon_{x} \circ \Upsilon_{y}\left(\exp \left({ }^{x} \frown^{y}\right)\right) \# \exp _{\#}\left(\frac{1}{2}\right\lrcorner_{x}\right) \# \exp _{\#}\left(\frac{1}{2}\right\lrcorner_{y}\right)
\end{aligned}
$$

so

$$
Z\left({ }_{x} \bigcirc \bigcirc_{y}\right)=\pi^{\mathrm{bc}} \Upsilon_{x} \circ \Upsilon_{y}\left(\exp \left({ }^{x} \frown^{y}\right)\right) .
$$

This is the first equality of Theorem 4. For the second equality,

$$
\hat{\Omega}_{y}\left(\exp \left({ }^{x} \frown^{y}\right)\right)=\Omega_{x} \sqcup \exp \left({ }^{x} \frown^{y}\right) .
$$

so

$$
Z\left({ }_{x} \mathrm{OO}_{y}\right)=\pi^{\mathrm{bc}} \Upsilon_{x}\left(\exp \left({ }^{y} \frown^{x}\right) \Omega_{x}\right) .
$$

For the last equality of the theorem, multiplicativity of $\Upsilon$ implies that

$$
\begin{aligned}
\pi^{\mathrm{bc}} \Upsilon_{x}\left(\exp \left({ }^{y} \frown^{x}\right) \Omega_{x}\right) & =\pi^{\mathrm{bc}}\left(\Upsilon_{x}\left(\exp \left({ }^{y} \frown^{x}\right)\right) \# \Upsilon_{x}\left(\Omega_{x}\right)\right) \\
& =\pi^{\mathrm{bc}}\left(\Upsilon_{x}\left(\exp \left({ }^{y} \frown^{x}\right)\right)\right) \# \chi\left(\Omega_{x}\right) \\
& =\chi\left(\exp \left({ }^{y} \frown^{x}\right) \sqcup \Omega_{y}\right) \# \chi\left(\Omega_{x}\right) .
\end{aligned}
$$

Hence we have

$$
Z\left({ }_{x}^{y}\right)=Z\left({ }_{x} \bigcirc_{y}\right) \# \Omega_{x}^{-1}=\exp \left({ }^{y} \frown^{x}\right) \sqcup \Omega_{y} .
$$

This completes the proof of Theorem 4 .

\section{Appendix}

To show that $\Omega^{\prime}=\Omega$, one can use the following "Sawon's identity [14]":

$$
\left\langle\Omega^{\prime},(\frown)^{n}\right\rangle=\left(\frac{1}{24} \ominus\right)^{n} .
$$

Geometry 8 Topology, Volume 7 (2003) 
Proof Proceed by induction on $n$. The result is trivial for $n=0$.

$$
\begin{aligned}
\left\langle\Omega^{\prime},(\frown)^{n}\right\rangle & =\left\langle\Omega^{\prime}, \frown \sqcup(\frown)^{n-1}\right\rangle & & \\
& =\left\langle\partial\left(\Omega^{\prime}\right),(\frown)^{n-1}\right\rangle & & \text { by Lemma } 4.2 \\
& =\frac{1}{24} \ominus\left\langle\Omega^{\prime},(\frown)^{n-1}\right\rangle & & \text { by Lemma } 5.1 \text { and explicit computation } \\
& =\left(\frac{1}{24} \ominus\right)^{n} & & \text { by induction }
\end{aligned}
$$

The following is well-known, see eg [8].

Lemma 6.4 In the Lie algebra $\mathfrak{s l}_{2}$, with the invariant inner product $\langle x, y\rangle=$ $-\operatorname{tr}(x y)$, where the trace is taken in the adjoint representation, we have the following relations:

$$
\bigcirc \equiv 3 \quad \Upsilon \equiv)(-X
$$

For example, apply the $\mathfrak{s} l_{2}$ relations, we find that that $\ominus \equiv 6$.

Lemma 6.5 Modulo the $\mathfrak{s} l_{2}$ relations, $\omega_{2 n} \equiv 2(\frown)^{n}$.

Proof Proceed by induction. This is a straightforward computation for $n=1$. For $n>1$, compute as follows:

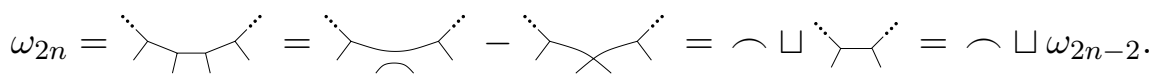

Lemma 6.6 Modulo the $\mathfrak{s l}_{2}$ relations, $\left\langle(\frown)^{n},(\frown)^{n}\right\rangle=(2 n+1)$ !.

Proof Proceed by induction. The statement is trivial for $n=0$. For $n>0$, the two ends of the first strut on the left hand side can either connect to the two ends of a single right hand strut or they can connect to two different struts. These happen in $2 n$ and $2 n \cdot(2 n-2)$ ways, respectively. (Note that there are $2 n \cdot(2 n-1)$ ways in all of gluing these two legs.) We therefore have

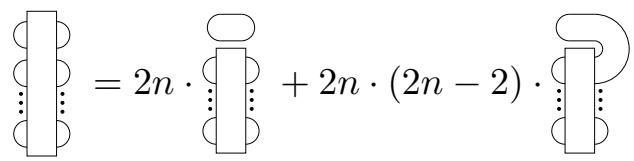

and

$$
\begin{aligned}
\left\langle(\neg)^{n},(\neg)^{n}\right\rangle & =(2 n \bigcirc+2 n \cdot(2 n-2))\left\langle\left(\neg^{n-1},(\neg)^{n-1}\right\rangle\right. \\
& \equiv 2 n \cdot(2 n+1)\left\langle\left(\frown^{n-1},(\neg)^{n-1}\right\rangle\right. \\
& \equiv(2 n+1) !
\end{aligned}
$$$$
\text { by induction. }
$$ 
Proposition 6.7 One has $\Omega^{\prime}=\Omega$.

Proof By Lemma 6.5, we find

$$
\Omega^{\prime}=\exp \left(\sum_{n} a_{2 n} \omega_{2 n}\right) \equiv \exp \left(\sum_{n} 2 a_{2 n}(\frown)^{n}\right) .
$$

Set $f(x)=\exp \left(2 \sum a_{2 n} x^{n}\right)=\sum f_{n} x^{n}$. Then by Lemma 6.6,

$$
\begin{aligned}
&\left\langle\Omega^{\prime},(\frown)^{n}\right\rangle \equiv\left\langle f(\frown),(\frown)^{n}\right\rangle=\left\langle f_{n}(\frown)^{n},(\frown)^{n}\right\rangle \equiv f_{n}(2 n+1) ! \\
&=\left(\frac{1}{24} \ominus\right)^{n} \equiv \frac{1}{4^{n}} \\
& f_{n}=\frac{1}{4^{n}(2 n+1) !} \\
& f(x)=\frac{\sinh (\sqrt{x} / 2)}{\sqrt{x} / 2} \\
& \exp \left(2 \sum_{n} a_{n} x^{n}\right)=\frac{\sinh (x / 2)}{x / 2} \\
& \sum_{n} a_{n} x^{n}=\frac{1}{2} \log \frac{\sinh (x / 2)}{x / 2} .
\end{aligned}
$$

so

\section{References}

[1] Anton Alekseev, Eckhard Meinrenken, The non-commutative Weil algebra, Invent. Math. 139 (2000) 135-172, arXiv:math.DG/9903052

[2] Martin Andler, Alexander Dvorsky, Siddhartha Sahi, Kontsevich quantization and invariant distributions on Lie groups, arXiv:math.QA/9910104

[3] Dror Bar-Natan, On the Vassiliev knot invariants, Topology 34 (1995) 423472

[4] Dror Bar-Natan, Non-associative tangles, from: "Geometric topology (Athens, GA, 1993)", AMS/IP Stud. Adv. Math. 2.1, Amer. Math. Soc. Providence, RI (1997) 139-183

[5] Dror Bar-Natan, Stavros Garoufalidis, Lev Rozansky, Dylan P Thurston, Wheels, wheeling, and the Kontsevich integral of the unknot, Israel J. Math. 119 (2000) 217-237, arXiv:q-alg/9703025

[6] Dror Bar-Natan, Stavros Garoufalidis, Lev Rozansky, Dylan P Thurston, The Aarhus integral of rational homology 3-spheres II: Invariance and universality, Selecta Mathematica, New Series, 8 (2002) 341-371, arXiv:math.QA/9801049 
[7] Dror Bar-Natan, Ruth Lawrence, A rational surgery formula for the LMO invariant, to appear in Israel J. Math. arXiv:math.GT/0007045

[8] Sergei V Chmutov, Alexander N Varchenko, Remarks on the Vassiliev knot invariants coming from $\mathrm{sl}_{2}$, Topology 36 (1997) 153-178

[9] Pierre Deligne, letter to Dror Bar-Natan, (January 1996) http://www.ma.huji.ac.il/〜drorbn/Deligne/

[10] Jacques Dixmier, Enveloping algebras, Graduate Studies in Mathematics 11, Amer. Math. Soc. Providence, RI (1996)

[11] Michel Duflo, Opérateurs différentiels bi-invariants sur un groupe de Lie, Ann. Scient. École Norm. Sup. 10 (1977) 265-288

[12] Roger Godement, Introduction à la théorie des groupes de Lie, Publications Mathématiques de l'Université Paris VII, Université de Paris VII, U.E.R. de Mathématiques, Paris, (1982)

[13] Vladimir Hinich, Arkady Vaintrob, Cyclic operads and algebra of chord diagrams, arXiv:math.QA/0005197

[14] Nigel Hitchin, Justin Sawon, Curvature and characteristic numbers of hyperkähler manifolds, Duke Math. J. 106 (2001) 599-615

[15] Maxim Kontsevich, Vassiliev's knot invariants, Advances in Soviet Mathematics 16 (1993) 137-150

[16] Maxim Kontsevich, Deformation quantization of Poisson manifolds, I, arXiv:q-alg/9709040

[17] Andrew Kricker, Bill Spence, Ian Aitchison, Cabling the vassiliev invariants, J. of Knot Theory and its Ramifications 5 (1996) 779-803

[18] Thang T Q Le, Jun Murakami, Representations of the category of tangles by Kontsevich's iterated integral, Commun. Math. Phys. 168 (1995) 535-562

[19] Thang T Q Le, Jun Murakami, The universal Vassiliev-Kontsevich invariant for framed oriented links, Compositio Math. 102 (1996) 41-64

[20] Thang T Q Le, Jun Murakami, Parallel version of the universal KontsevichVassiliev invariant, J. Pure and Appl. Alg. 212 (1997) 271-291

[21] Thang T Q Le, Jun Murakami, Tomotada Ohtsuki, On a universal perturbative invariant of 3-manifolds, Topology 37-3 (1998) 539-574

[22] Jens Lieberum, On Vassiliev invariants not coming from semisimple Lie algebras, J. of Knot Theory and its Ramifications 5 (2000) 275-299, arXiv: math.QA/9806064

[23] Takuro Mochizuki, On the morphism of Duflo-Kirillov type, J. Geom. Phys. 41 (2002) 73-113

[24] Jun Murakami, Tomotada Ohtsuki, Topological quantum field theory for the universal quantum invariant, Commun. Math. Phs. 188 (1997) 501-520

[25] Dylan Thurston, Torus actions for the LMO invariant, in preparation

[26] Pierre Vogel, Algebraic structures on modules of diagrams, Tech. report, Université Paris VII (July 1995) 